Pacific Journal of Mathematics

FIXED POINTS IN A TRANSFORMATION GROUP 


\title{
FIXED POINTS IN A TRANSFORMATION GROUP
}

\section{HsIN CHU}

\begin{abstract}
In this paper, the following result is proved: "Let $(X, T, \pi)$ be a transformation group, where $X$ is a Peano continuum with an end point fixed under $T$. If the group $T$ is one of the following two types: (1) It contains a subgroup $R^{n}$ such that $G / R^{n}$ is compact or $(2)$ It contains a s subgroup $Z \cdot R^{n}$ such that $G /\left(Z \cdot R^{n}\right)$ is compact, where $Z$ is isomorphic to the discrete additive group of all integers, then $T$ has another fixed point."
\end{abstract}

Professor A. D. Wallace, in [4], proved the following: "Let $(X, Z, \pi)$ be a transformation group, where $Z=$ the discrete additive group of all integers. If $X$ is a Peano continuum with a fixed end point under $Z$, then $Z$ has another fixed point." An interesting question, (See [5]) has been raised by Wallace: "Can one reach the same conclusion about either compact groups or abelian groups"? In the case of compact groups, Professor H. C. Wang answered the question in the affirmative (See [6]). We also give an affirmative answer to the question in the case of abelian groups when the abelian group is of the type either $R^{n} \cdot K$ or $Z \cdot R^{n} \cdot K$ where $R^{n}$ is a vector group of dimension $n$ and $k$ is a compact abelian group. Actually, we also cover the case of non-abelian groups. The same conclusion can be reached if the group, $G$, is one of the following two types:

(1) It contains a subgroup $R^{n}$ such that $G / R^{n}$ is compact or

(2) It contains a subgroup $Z \cdot R^{n}$ such that $G /\left(Z \cdot R^{n}\right)$ is compact.

2. We divide that proof of our main result into several steps.

Lemma 1. Let $(X, T, \pi)$ be a transformation group, where $X$ is an arcwise connected Hausdorff space with an end point $e$ fixed under T. If $X$ has a closed invariant set $A$ under $T$ which does not contain $e$ then $T$ has another fixed point. Let $1(t), 0 \leqq t \leqq 1$, be an arc connecting $e$ and some point $x$ in $A$ such that $1(0)=e$ and $1(1)=x$. All the points which separate $e$ and $A$ lie on $1(t)$. Let $S$ be the set of all those points. $S$ is not empty. Introduce a linear ordering in $1(t), 0 \leqq t \leqq 1$, by the natural linear ordering of $t$. Then the upper limit point of $S$ is a fixed point, other than $e$, under $T$.

Proof. The first part of the lemma is an equivalent statement of a theorem, in [6], of Professor H. C. Wang. Under the same assumption

Received June 8, 1964. This work was supported by Contract NAS8-1646, with the George C. Marshall Space Flight Center, NASA, Huntsville, Alabama. 
as our lemma, Wang's conclusion is that $T$ has no other fixed point if and only if, given any neighborhood $U$ of $e$, the orbit $U T$ under $T$ coincides with the whole space $X$. We notice that if $S$ is a closed invariant set under $T$ which does not contain $e$, then $U=X-S$ is a neighborhood of $e$ and $U T=U$ which does not coincide with the whole space $X$ and vice versa.

The proof of the second part of this lemma can be obtained from the proof of Wang's theorem. (See [6]).

Lemma 2. Let $(X, Z, \pi)$ be a transformation group. If $X$ is a compact, connected, Hausdorff space which is more than a point and has a fixed end point $e$, then there is a closed set $H \subset X-e$, which is invariant under $Z$.

Proof. This is a theorem by Wallace, See [4].

By Lemma 1 and Lemma 2, we obtain Wallace's result.

LeMma 3. Let $(X, Z, \pi)$ be a transformation group. If $X$ is a Peano continuum with a fixed end point e under $Z$, then $Z$ has another fixed point.

LEMMA 4. Let $(X, T, \pi)$ be a transformation group. If $X$ is a Peano continuum with a fixed end point $e$ under $T$ and $T$ contains a syndetic subgroup $Z$ (i.e. $T$ contains a integer group $Z$ such that $T / Z$ is a compact set), then $T$ has another fixed point. If, furthermore, $T$ is connected, then the assumption on the given end point being fixed under $T$ is not necessary.

Proof. Consider the transformation group $(X, Z, \pi)$ induced by $(X, T, \pi)$. From Lemma 3, we know that there is another fixed point $p$ under $Z$. Since $Z$ is syndetic, there is a compact subset $K$ in $T$ such that $T=Z \cdot K$. Consequently, $p T=(p Z) K=p K$ which is compact and therefore, is closed. It is clear that $e \notin p K$. We know $p K$ is closed and invariant under $T$. By Lemma $1, X$ has another fixed point $q$ under $T$.

If $T$ is connected, it is easy to see that every end point is fixed under $T$ (See [5]). Suppose $e$ is an end point and $e \neq e t$ for some $t \in T$. Then, because $e$ is an end point and $e T$ is connected, there is $s \in e T$ such that $s$ separates $e$ and $e t$. Consequently, there exists some $t^{\prime} \in T$ such that $s=e t^{\prime}$. It follows that as $t^{\prime}$ is a homeomorphism of $X$, et is also an end point as well as a cut point. A contradiction!

As a direct consequence of Lemma 4, we have: 
Lemma 5. Let $(X, R, \pi)$ be a transformation group. If $X$ is a Peano continuum with an end point, then $R$ has another fixed point.

Lemma 6. Let $\left(X, R^{n}, \pi\right)$ be a transformation group where $n$ is a positive integer. If $X$ is a Peano continuum with an end point $e$, then $R^{n}$ has another fixed point.

Proof. By Lemma 4, we know that the end point $e$ is fixed under $R^{n}$ for all $n$. The proof of this lemma is by induction. Suppose the statement is true for $n=k$. Consider $n=k+1$. Let $\left(x_{1}, \cdots, x_{k}, x_{k+1}\right)$ be a coordinate system of $R^{k+1}$. Let $A$ and $B$ be the closed subgroups determined by $x_{1}=0$ and $x_{2}=0$ respectively. Then $A \cong B \cong R^{k}$. Let the transformation groups $(X, A, \pi)$ and $(X, B, \pi)$ both be induced by $\left(X, R^{k+1}, \pi\right)$. By the inductive assumption, we know there are two points $p$ and $q$ such that $p$ is invariant under $A$ and $q$ is invariant under $B$. Both $p$ and $q$ are distinct from $e$. Let $C_{1}$ be the subgroup of $R^{k+1}$ determined by $x_{2}=0, \cdots, x_{k+1}=0$. Let $C_{2}$ be the subgroup of $R^{k+1}$ determined by $x_{1}=0, x_{3}=0, \cdots, x_{k+1}=0$. Then $C_{1} \cong C_{2} \cong R$ and, as direct products $R^{k+1}=C_{1} \cdot A=C_{2} \cdot B$. Consider the orbit, $(p) R^{k+1}$, of $p$ under $R^{k+1}$ and the orbit, $(q) R^{k+1}$, of $q$ under $R^{k+1}$. It is clear that $(p) R^{k+1}=(p) C_{1}$ and $(q) R^{k+1}=(q) C_{2}$, where $(p) C_{1}$ and $(q) C_{2}$ both are connected.

We know both $\operatorname{cl}\left((p) C_{1}\right)$ and $\operatorname{cl}\left((q) C_{2}\right)$ are invariant under $R^{k+1}$. If $e$ is not in either $\operatorname{cl}\left((p) C_{1}\right)$ or $\operatorname{cl}\left((q) C_{2}\right)$, then, by Lemma $1, R^{k+1}$ has another fixed point. Suppose $e$ is in both $\operatorname{cl}\left((p) C_{1}\right)$ and $\operatorname{cl}\left((q) C_{2}\right)$. This implies that every neighborhood of $e$ contains points from both $(p) C_{1}$ and $(q) C_{2}$.

Let $U_{e}$ be a neighborhood of $e$ such that $\{p, q\} \cap U_{e}=\phi$. Since $e$ is a fixed end point, there exists $x \in U_{e}$ such that $X-x=X_{1} \cup X_{2}$ for some sets $X_{1}$ and $X_{2}$ with the properties:

$$
X_{1} \cap \operatorname{cl}\left(X_{2}\right)=\operatorname{cl}\left(X_{1}\right) \cap X_{2}=\phi \quad \text { and } e \in X_{1} \subset U_{e} .
$$

Consequently, $\{p, q\} \subset X_{2}$. Notice that $X_{1}$ is open in $X$. It follows that $X_{1}$ contains points from both $(p) C_{1}$ and $(q) C_{2}$. Since both $(p) C_{1}$ and $(q) C_{2}$ are connected, it follows that $x \in(p) C_{1} \cap(q) C_{2}$. Since $R^{k+1}$ is abelian, we have $p=q$ and $p$ is a fixed point under $R^{k+1}$ other than $e$. Complete the proof by Lemma 5 .

LeMma 7. Let $\left(X, Z \cdot R^{n}, \pi\right)$ be a transformation group. If $X$ is a Peano continuum with a fixed end point e under $Z \cdot R^{n}$, then $Z \cdot R^{n}$ has another fixed point.

Proof. If $n=0$, the statement of this lemma is the same as Lemma 3. Let $n>0$. Let $(X, A, \pi)$ be a transformation group induced 
by $\left(X, Z \cdot R^{n}, \pi\right)$ where $A=Z \cdot R^{n-1}$ is a subgroup of $Z \cdot R^{n}$. Let $B \cong R$ be a subgroup of $Z \cdot R^{n}$ such that $Z \cdot R^{n}=A \cdot B$. Prove this lemma by induction on $n$. Suppose $(X, A, \pi)$ has a fixed point, $p$, other than $e$, under $A$. Consider the orbit $(p)\left(Z \cdot R^{n}\right)$. It is clear that $(p)\left(Z \cdot R^{n}\right)=$ $(p) B$, which is connected. The orbit-closure $\operatorname{cl}\left((p)\left(Z \cdot R^{n}\right)\right)$ is a connected compact Hausdorff space. Obviously, $\operatorname{cl}\left((p)\left(Z \cdot R^{n}\right)\right)$ is invariant under $Z \cdot R^{n}$. If $e$ is not in $c l\left((p)\left(Z \cdot R^{n}\right)\right)$, then, by Lemma $1, Z \cdot R^{n}$ has another fixed point. Suppose $e \in \operatorname{cl}\left((p)\left(Z \cdot R^{n}\right)\right)$. Let $Z^{\prime}$ be an integer group of $B$. Then $e$ is a fixed end point of the transformation group $\left(\operatorname{cl}\left((p)\left(Z \cdot R^{n}\right)\right), Z^{\prime}, \pi\right)$. By Lemma 2 , there is a $Z^{\prime}$-invariant closed subset $H$ of $\operatorname{cl}\left((p)\left(Z \cdot R^{n}\right)\right)$ such that $e \notin H$. Consider the transformation group $\left(X, Z^{\prime}, \pi\right)$, induced by $\left(X, Z \cdot R^{n}, \pi\right)$. Choose a point $q \in H$ and connect $e$ and $q$ by an arc $1(t), 0 \leqq t \leqq 1$ on which $1(0)=e$ and $1(1)=q$. Let $S$ be the set of all points which separate $e$ and $H$. By Lemma 1 the upper limit point, $r$, of $S$ is a fixed point, other than $e$, under $Z^{\prime}$. Since $\operatorname{cl}\left((p)\left(Z \cdot R^{n}\right)\right)$ is connected, we have $S \subset \operatorname{cl}\left((p)\left(Z \cdot R^{n}\right)\right)$. Consequently, $r \in \operatorname{cl}\left((p)\left(Z \cdot R^{n}\right)\right)$. Since the points in $(p)\left(Z \cdot R^{n}\right)$ are fixed under $A$, the points in $\operatorname{cl}\left((p)\left(Z \cdot R^{n}\right)\right)$ are also fixed under $A$. It follows that $r$ is fixed under both $A$ and $Z^{\prime}$. Let $B=Z^{\prime} K^{\prime}$ for some compact set $K^{\prime}$. Then $(r)\left(Z \cdot R^{n}\right)=(r) K^{\prime}$ which is compact. It is obvious $e \notin(r) K^{\prime}$. By Lemma $1,\left(Z \cdot R^{n}\right)$ has another fixed point. Complete the proof by induction.

THEOREM. Let $(X, T, \pi)$ be a transformation group. If $X$ is a Peano continuum with a fixed end point under $T$ and $T$ is one of the following two types:

(1) It contains a subgroup $R^{n}$ such that $G / R^{n}$ is compact or

(2) It contains a subgroup $Z \cdot R^{n}$ such that $G / Z \cdot R^{n}$ is compact.

Proof. Complete the proof by Lemma 1, Lemma 6, Lemma 7 and a similar method used in the proof of Lemma 4 .

CoRollary 1. Let $(X, T, \pi)$ be a transformation group. If $X$ is a Peano continuum with an end point and $T$ is locally compact, connected, abelian group, then $T$ has another fixed point.

We have the following application in Topological Dynamics. (See [1]). The proof is similar to the one used for the theorem.

Corollary 2. Let $(X, T, \pi)$ be a transformation group. If $X$ is arcwise connected, Hausdorff with a fixed end point $e$ and a regularly almost periodic point $p$, other than $e$, then $T$ has another fixed point.

Proof. By the definition of regularly almost periodic point, for a closed neighborhood $U$ of $p$ such that $e \notin U$, there exists a syndetic 
subgroup $A$ of $T$ such $p A \subset U$. It follows that $\operatorname{cl}(p A) \subset U$, and thereby, $e \notin c l(p A)$. It is clear that $\operatorname{cl}(x A)$ is invariant under $A$. By Lemma 1, we have another fixed point $q$ under $A$. Since $A$ is syndetic, there exists a compact set $K$ such that $T=A \cdot K$. From $q T=(q A) K=q K$, we know $q T$ is compact and, therefore, is closed and $e \notin q T$. Since $q T$ is invariant under $T$, by Lemma 1 we have another fixed point under $T$. The theorem is proved.

\section{REFERENCES}

1. W. H. Gottschalk and G. A. Hedlund, Topological dynamics, Amer. Math. Soc. Colloq. Publ., Vol. 36, Amer. Math. Soc., Providence, R. I. (1955).

2. D. Montgomery and L. Zippin, Transformation, groups, Interscience, New York, (1955).

3. L. Pontrjagin, Topological groups, Princeton, University. Press, (1939).

4. A. D. Wallace, A fixed-point theorem, Bull. Amer. Math. Soc. 51 (1945), 413-416.

5. - Group invariant continua, Fund. Math. 36 (1949), 119-124.

6. H. C. Wang, A remark on transformation group leaving fixed an end point, Proc. Amer. Math. Soc. 3 (1952), 548-549.

7. R. L. Wilder, Topology of manifolds, Amer, Math. Soc. Colloq. Publ., Vol. 32, Amer. Math. Soc., Providence, R. I., (1949).

University of Alabama Research Institute 



\title{
PACIFIC JOURNAL OF MATHEMATICS
}

\author{
EDITORS
}

\author{
H. SAmelson \\ Stanford University \\ Stanford, California \\ R. M. Blumenthal \\ University of Washington \\ Seattle, Washington 98105
}

\author{
J. DugundjI \\ University of Southern California \\ Los Angeles, California 90007
}

*Richard Arens

University of California

Los Angeles, California 90024

\section{ASSOCIATE EDITORS}
E. F. BECKENBACH
B. H. NeUmanN
F. WOLF
K. YoSIDA

\section{SUPPORTING INSTITUTIONS}

\author{
UNIVERSITY OF BRITISH COLUMBIA \\ CALIFORNIA INSTITUTE OF TECHNOLOGY \\ UNIVERSITY OF CALIFORNIA \\ MONTANA STATE UNIVERSITY \\ UNIVERSITY OF NEVADA \\ NEW MEXICO STATE UNIVERSITY \\ OREGON STATE UNIVERSITY \\ UNIVERSITY OF OREGON \\ OSAKA UNIVERSITY \\ UNIVERSITY OF SOUTHERN CALIFORNIA
}

\author{
STANFORD UNIVERSITY \\ UNIVERSITY OF TOKYO \\ UNIVERSITY OF UTAH \\ WASHINGTON STATE UNIVERSITY \\ UNIVERSITY OF WASHINGTON \\ * * * * \\ AMERICAN MATHEMATICAL SOCIETY \\ CALIFORNIA RESEARCH CORPORATION \\ SPACE TECHNOLOGY LABORATORIES \\ NAVAL ORDNANCE TEST STATION
}

Mathematical papers intended for publication in the Pacific Journal of Mathematics should by typewritten (double spaced). The first paragraph or two must be capable of being used separately as a synopsis of the entire paper. It should not contain references to the bibliography. No separate author's resumé is required. Manuscripts may be sent to any one of the four editors. All other communications to the editors should be addressed to the managing editor, Richard Arens, at the University of California, Los Angeles, California 90024.

50 reprints per author of each article are furnished free of charge; additional copies may be obtained at cost in multiples of 50 .

The Pacific Journal of Mathematics is published quarterly, in March, June, September, and December. Effective with Volume 13 the price per volume (4 numbers) is $\$ 18.00$; single issues, $\$ 5.00$. Special price for current issues to individual faculty members of supporting institutions and to individual members of the American Mathematical Society: $\$ 8.00$ per volume; single issues $\$ 2.50$. Back numbers are available.

Subscriptions, orders for back numbers, and changes of address should be sent to Pacific Journal of Mathematics, 103 Highland Boulevard, Berkeley 8, California.

Printed at Kokusai Bunken Insatsusha (International Academic Printing Co., Ltd.), No. 6, 2-chome, Fujimi-cho, Chiyoda-ku, Tokyo, Japan.

PUBLISHED BY PACIFIC JOURNAL OF MATHEMATICS, A NON-PROFIT CORPORATION

The Supporting Institutions listed above contribute to the cost of publication of this Journal, but they are not owners or publishers and have no responsibility for its content or policies.

* Basil Gordon, Acting Managing Editor until February 1, 1966. 


\section{Pacific Journal of Mathematics}

\section{Vol. 15, No. $4 \quad$ December, 1965}

Robert James Blattner, Group extension representations and the structure space ........... 1101

Glen Eugene Bredon, On the continuous image of a singular chain complex .............. 1115

David Hilding Carlson, On real eigenvalues of complex matrices .................... 1119

Hsin Chu, Fixed points in a transformation group ............................. 1131

Howard Benton Curtis, Jr., The uniformizing function for certain simply connected Riemann

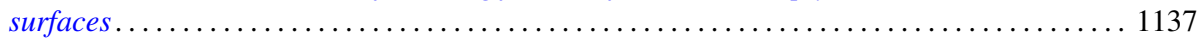

George Wesley Day, Free complete extensions of Boolean algebras................... 1145

Edward George Effros, The Borel space of von Neumann algebras on a separable Hilbert

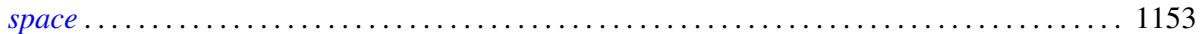

Michel Mendès France, $A$ set of nonnormal numbers ......................... 1165

Jack L. Goldberg, Polynomials orthogonal over a denumerable set ................ 1171

Frederick Paul Greenleaf, Norm decreasing homomorphisms of group algebras . . . . . . . . 1187

Fletcher Gross, The 2-length of a finite solvable group ........................ 1221

Kenneth Myron Hoffman and Arlan Bruce Ramsay, Algebras of bounded sequences ........ 1239

James Patrick Jans, Some aspects of torsion . . . . . . . . . . . . . . . . . . . . . . . 1249

Laura Ketchum Kodama, Boundary measures of analytic differentials and uniform

approximation on a Riemann surface ............................... 1261

Alan G. Konheim and Benjamin Weiss, Functions which operate on characteristic

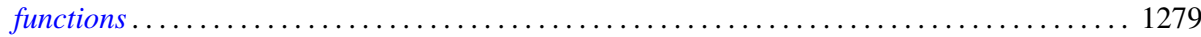

Ronald John Larsen, Almost invariant measures ............................ 1295

You-Feng Lin, Generalized character semigroups: The Schwarz decomposition ............ 1307

Justin Thomas Lloyd, Representations of lattice-ordered groups having a basis . . . . . . . . 1313

Thomas Graham McLaughlin, On relative coimmunity ....................... 1319

Mitsuru Nakai, $\Phi$-bounded harmonic functions and classification of Riemann surfaces ....... 1329

L. G. Novoa, On n-ordered sets and order completeness ..................... 1337

Fredos Papangelou, Some considerations on convergence in abelian lattice-groups . . . . . . . 1347

Frank Albert Raymond, Some remarks on the coefficients used in the theory of homology

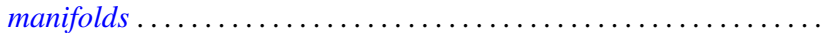

John R. Ringrose, On sub-algebras of a $C^{*}$-algebra .

Jack Max Robertson, Some topological properties of certain spaces of differentiable

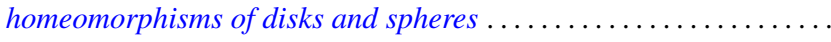

Zalman Rubinstein, Some results in the location of zeros of polynomials

Arthur Argyle Sagle, On simple algebras obtained from homogeneous general Lie triple systems. . . .

Hans Samelson, On small maps of manifolds ............................... 1401

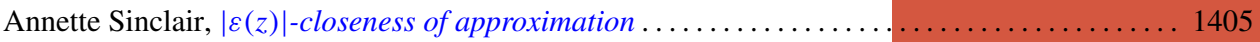

Edsel Ford Stiel, Isometric immersions of manifolds of nonnegative constant sectional curvature

Earl J. Taft, Invariant splitting in Jordan and alternative algebras ................. 1421

L. E. Ward, On a conjecture of R. J. Koch . . . . . . . . . . . . . . . . . . . . . . . . . . . 1429

Neil Marchand Wigley, Development of the mapping function at a corner . . . . . . . . . . 1435

Horace C. Wiser, Embedding a circle of trees in the plane ....................... 1463

Adil Mohamed Yaqub, Ring-logics and residue class rings . . . . . . . . . . . . . . . . 1465

John W. Lamperti and Patrick Colonel Suppes, Correction to: Chains of infinite order and their application to learning theory ........................................ 1471

Charles Vernon Coffman, Correction to: Non-linear differential equations on cones in Banach

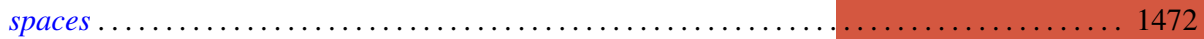

P. H. Doyle, III, Correction to: A sufficient condition that an $\operatorname{arc}$ in $S^{n}$ be cellular. . . . . . . . 1474

P. P. Saworotnow, Correction to: On continuity of multiplication in a complemented algebra 